Muséologies

Les cahiers d'études supérieures

muséologies

\title{
Documenter et communiquer la recherche en arts numériques par la mise en situation dialogale de l'artiste contemporain
}

\section{Stéphane Bellin}

Volume 7, numéro 1, 2014

Le dialogue dans les musées d'art contemporain

URI : https://id.erudit.org/iderudit/1026655ar

DOI : https://doi.org/10.7202/1026655ar

Aller au sommaire du numéro

Éditeur(s)

Association Québécoise de Promotion des Recherches Étudiantes en

Muséologie (AQPREM)

ISSN

1718-5181 (imprimé)

1929-7815 (numérique)

Découvrir la revue

Citer cet article

Bellin, S. (2014). Documenter et communiquer la recherche en arts numériques par la mise en situation dialogale de l'artiste contemporain. Muséologies, 7(1), 209-223. https://doi.org/10.7202/1026655ar

Tous droits réservés ( Association Québécoise de Promotion des Recherches Étudiantes en Muséologie (AQPREM), 2014
Ce document est protégé par la loi sur le droit d'auteur. L’utilisation des services d'Érudit (y compris la reproduction) est assujettie à sa politique d'utilisation que vous pouvez consulter en ligne.

https://apropos.erudit.org/fr/usagers/politique-dutilisation/ 
Article neuf

\section{Documenter et communiquer la} recherche en arts numériques par la mise en situation dialogale de l'artiste contemporain

Stéphane Bellin 
Après avoir réalisé des études en histoire de l'art, Stéphane Bellin a complété un master professionnel dans les métiers de l'art contemporain. Aujourd'hui, il poursuit son parcours universitaire en entreprenant une thèse de doctorat en muséologie sur les dispositifs documentaires, comme forme de communication de la recherche en arts numériques, sous la direction des professeures Cécile Tardy, de l'Université d'Avignon et des Pays du Vaucluse, et Johanne Lalonde, de l'Université du Québec à Montréal.

stef.bellin@netcourrier.com 
La recherche en arts numériques est une activité profondément hybride, qui se caractérise par sa capacité à produire simultanément une œuvre, des dispositifs techniques et des connaissances théoriques sur l'art et les technologies ${ }^{1}$. La multiplicité de ses enjeux et l'éclatement de ses résultats font qu'ils sont valorisés dans des champs disciplinaires différents et par de multiples contributeurs ${ }^{2}$. De plus, dans le contexte de l'université ou des écoles supérieures d'art, de nombreux artistes-chercheurs ont relevé que la recherche en arts numériques ne se donne pas immédiatement dans ses résultats, car elle repose en grande partie sur des pratiques difficilement observables. Concrètement, sur le terrain, le mémoire universitaire s'est généralisé, incitant les étudiants, au-delà de leurs productions plastiques, à écrire. Certains enseignants refusent cette forme de production et de construction des savoirs de la recherche en arts numériques, jugeant que sa forme est trop normative, académique et non appropriée à ses spécificités. Si, pendant longtemps, seule l'œuvre d'art valait comme résultat de recherche, aujourd'hui les écoles d'art considèrent que la recherche en arts numériques est également productive de savoirs transdisciplinaires, qu'il est désormais nécessaire d'enregistrer pour être en mesure de les transmettre et les communiquer à autrui. Aussi, partagée entre théorie de l'art et pratique artistique, la recherche en arts numériques cherche-t-elle à inventer les modalités de sa communication scientifique. Son articulation entre la théorie et la pratique induit l'émergence d'une communication mobilisant une parole à modeler qui, selon Pierre Gosselin, professeur dans le contexte québécois de la recherche création, se situerait dans un entredeux, entre le faire de la pratique créative et le

1 FOURMENTRAUX, Jean-Paul. "L'art à l'épreuve de la recherche. Profession et identité artistiques en mutation ". In. BIANCHINI, Samuel (dir.). Recherche et création. Nancy: Éditions du Parc, 2009, p. 80.

2 FOURMENTRAUX, Jean-Paul. Artistes de laboratoire,

Recherche et création à l'ère numérique. Paris: Hermann, 2011, p. 75 .

3 BURNS, L. Sophia. "La parole de l'artiste chercheur ". In. GOSSELIN, Pierre et Éric LE COEGUIEC (dir.). La Recherche création, pour une compréhension de la recherche en pratique artistique. Québec: Presses de l'Université du Québec, 2006, p. 63. verbe de la pratique théorique de la recherche ${ }^{3}$. L'argumentaire de cet article repose sur ce premier postulat que les formes de communications scientifiques dans le domaine de la recherche en art ne permettent pas d'en restituer toutes les connaissances produites.

Directeur de l'École supérieure d'art et design de Saint-Étienne, Emmanuel Tibloux affirme que le mémoire dans les écoles d'art " pour peu que sa forme ne soit pas contrainte par des normes trop académiques [...] peut être aussi bien le moyen de situer ou documenter un travail artistique que l'occasion de mettre en œuvre, dans une écriture singulière, une approche plastique de la langue, définition possible de la poésie $"{ }^{4}$. Dans le domaine des arts numériques, l'artiste et chercheur Samuel Bianchini va plus loin et pose le constat de la nécessité, dans le domaine de la recherche en art, d'élaborer des communications scientifiques, qui reposent sur une écriture qui se concentre d'abord sur les processus de création plutôt que sur les résultats ${ }^{5}$. Ces deux constats aboutissent à un second postulat qui consiste à penser que la communication scientifique dans la recherche en arts numériques pourrait prendre la forme d'une documentation du processus créatif, conçue comme un ensemble de documents qui comprendraient des informations sur ce dernier, associés et organisés de manière à rendre compte des savoirs qui y sont développés. Ce glissement potentiel de la documentation vers la communication scientifique constitue l'hypothèse qui régit l'assise du présent article.

Porteuse de l'information scientifique et technique, la communication scientifique se caractérise par l'énonciation des connaissances

4 TIBLOUX, Emmanuel. "Écoles d'art: nouveaux enjeux ". ArtPress 2, no 22, 2011, p. 15.

5 BIANCHINI, Samuel. "Recherche et création ». In. BIANCHINI, Recherche et création, op. cit., p. 37. 
et des savoir-faire émanant des activités scientifiques et techniques. Aujourd'hui, dans la plupart des sciences, le savoir est mis en discours sous la forme du texte, plus précisément de l'article dans la revue scientifique, la recherche scientifique ayant pour objectif, selon la formule du chercheur Yves Jeanneret, d' "écrire la science " ${ }^{6}$. Ce dernier précise même: " écrire la science, c'est tenir un discours, qui dialogue avec d'autres discours " ${ }^{7}$. Cependant, le geste et la parole ont été les premiers véhicules de transfert des connaissances ${ }^{8}$. Cette diffusion directement d'homme à homme existe encore aujourd'hui sous la forme de réunions de travail, de journées d'étude, de colloques, de conférences ou encore de forums. Analysé selon une perspective historique, l'usage de l'écriture s'est accru pour " consigner ou enregistrer sur un support ce qu'il (un individu) détient dans sa mémoire individuelle et d'accumuler au fil des ans (les) savoir(s) et savoir-faire de la communauté $"^{9}$. Les publications scientifiques, qu'il s'agisse des articles, des thèses, des rapports ou des livres écrits par les chercheurs, sont encore les documents les plus courants en termes de diffusion des nouvelles connaissances. En sciences de l'art, la communication scientifique prend principalement la forme de l'exposition et du catalogue. L'exposition est présentée par Joëlle Le Marec et Roland Topalian comme une mise en situation d'énonciation plurielle. Elle est conçue alors comme " un objet textuel au sens large, c'est-à-dire un objet associant texte, image, son et pouvant tout à la fois être lu, vu et parcouru. Sa dimension polyphonique peut être analysée en termes d'énonciation plurielle ${ }^{10}$. Mais il existe sans doute d'autres formes de mise en situation d'énonciation dans lesquelles le savoir pourrait s'investir, comme l'écrit Yves Jeanneret:

6 JEANNERET, Yves. Écrire la science: formes et enjeux de la vulgarisation. Paris: Presses universitaires de France, 1994, p. 7. 7 Ibid.

8 COMBREROUSSE, Martine. Histoire de l'information scientifique et technique. Paris : Nathan, 1999.

9 CACALY, Serge. Dictionnaire de l'information, Paris: Armand Colin, 2008, p. 77.

10 LE MAREC, Joëlle et Roland TOPALIAN. «Énonciation plurielle et publication de la parole du public en contexte
Les sources de l'information scientifique et technique sont tributaires de ceux qui élaborent les savoirs: savants, chercheurs, érudits, ingénieurs... Les connaissances en tant que résultats de l'acte de connaître, sont alors communiquées ou conservées sous des formes diverses, adaptées aux usages envisagés ${ }^{11}$.

Au regard de ces ajustements sur la nature de la communication scientifique, le présent article tente de répondre à cette problématique qui consiste à savoir si la documentation, dans le domaine de la recherche en arts numériques, est porteuse d'une énonciation productive de savoir scientifique.

\section{Interroger le dispositif documentaire de Kawenga, territoires numériques comme situation d'énonciation}

Le cadre général d'analyse examine la documentation comme situation d'énonciation, c'est-à-dire qu'il s'agit d'étudier sous quelle logique d'énonciation est structuré le dispositif documentaire observé. Dépassant une simple analyse formelle de celui-ci, cet article tente de proposer une analyse orientée vers une vision de la documentation comme espace de production de langage. Celle-ci est alors perçue comme un « objet textuel large » issu d'une " écriture ».

L'analyse de la documentation comme situation d'énonciation mobilise deux conceptsoutils empruntés aux sciences de l'information et de la communication: 1) le concept de dispositif info-communicationnel ${ }^{12}$, tel qu'il est défini par Viviane Couzinet, qui permet de l'examiner sous ses différentes dimensions (technique, socio-organisationnelle et socio-économique) et 2) celui d'énonciation

muséal: le cas de la tribune des visiteurs ". Communication et langages, 2003, vol. 135, no 135, p. 12.

11 JEANNERET, Écrire la science: formes et enjeux de la vulgarisation, op.cit. p. 7.

12 COUZINET, Viviane. "Dispositif info-communicationnels : contributions à une définition ". In. COUZINET, Viviane (dir.). Dispositifs info-communicationnels, questions de médiations documentaires. Paris : Lavoisier, 2009, p. 19-29. 
éditoriale ${ }^{13}$, dont la délimitation est attribuée à Emmanuel Souchier, qui permet de concevoir son énonciation polyphonique. Dès lors, étudier la documentation, comme dispositif info-communicationnel, consiste à déterminer ses acteurs, les documents et les types d'informations transmises, le contexte duquel émane le dispositif de documentation, le moment où il est exécuté, les techniques qui le matérialisent et enfin son intentionnalité. En ce sens, l'étude de la documentation comme dispositif info-communicationnel facilite sa compréhension en tant que situation d'énonciation documentaire (Qui parle? À qui ? Quels types d'informations? Quand? Où? Avec quelles intentions ?). Ce genre d'étude fournit les éléments qui permettent, dans un second temps, la mise en évidence des marques de cette énonciation documentaire et qui mettent donc en exergue les processus communicationnels qui la régissent.

La présente étude s'appuie sur un travail de recherche, pas uniquement basé sur l'observation et la théorie, mais également sur l'expérimentation et la participation, celles d'une expérience de praticien-chercheur. En effet, cet article se fonde sur un projet de recherche qui s'est réalisé dans le cadre d'une convention CIFRE $^{14}$ réunissant Kawenga, territoires numériques et un doctorant - moi-même de l'équipe Culture et Communication du laboratoire du Centre Norbert Elias (Université d'Avignon et des Pays du Vaucluse). Le travail exposé ici émane à la fois de l'activité réflexive théorique propre à la position d'un jeune chercheur et de la possibilité qui lui a été offerte d'expérimenter et d'inventer un dispositif documentaire au sein d'une structure culturelle. Cette recherche en CIFRE a induit un positionnement particulier par rapport à l'objet d'étude dans le sens où le chercheur est

13 SOUCHIER, Emmanuel. "L'image du texte, pour une théorie de l'énonciation éditoriale ". Les cahiers de médiologie, no 6,1998 , p. 137-145.

14 La convention CIFRE (Convention industrielle de formation par la recherche) est un dispositif financier du ministère de l'Enseignement supérieur et de la Recherche subventionnant toute entreprise ou structure de droit français qui embauche un doctorant pour le placer au cœur d'une collaboration de recherche avec un laboratoire public universitaire. devenu acteur du terrain étudié. Cette posture est proche des pratiques de la recherche participative ${ }^{15}$, qui réfutent l'idée générale d'une recherche appartenant à un monde à part, imposant au chercheur de se situer " éloigné de son terrain ". Dès lors, cet article fait état d'une recherche profondément ancrée dans les réalités d'un contexte institutionnel, celui de Kawenga, territoires numériques, et dans la pratique fusionnant la logique d'action à celle de production de savoir. Si ce positionnement se rapproche en de nombreux points de la démarche d'une recherche participative, la posture adoptée dépasse cette dernière; la posture de praticien fusionne ici avec celle de chercheur. Par ailleurs, la posture de recherche adoptée consiste à dépasser une pratique de praticien de la documentation pour en dégager du sens et du savoir par la mise en place et le déploiement d'un processus de recherche réflexif. Une telle démarche de recherche par l'action induit un engagement du chercheur le situant simultanément dans la pratique et dans l'analyse, le but étant d'améliorer la pratique expérimentée. Cette volonté rejoint une des caractéristiques principales de la rechercheaction, celle de provoquer un changement dans la réalité étudiée : "Elle cherche à corriger, infléchir ou transformer la réalité [...] Ainsi, la recherche-action vise-t-elle avant tout à optimiser l'action ${ }^{16}$."

\section{Le contexte: Kawenga, territoires numériques, un medialab artistique ${ }^{17}$}

L'association culturelle Kawenga, territoires numériques, appartient au domaine de la recherche en arts numériques à travers son approche de medialab artistique. Un concept qui s'inspire du modèle du MIT Media Lab, créé en 1985 au Massachusetts Institute of

15 La notion de recherche participative est considérée dans son acceptation la plus large, c'est-à-dire qu'elle englobe les différentes variations de la recherche-action 16 RESWEBBER, Jean-Paul. La recherche-action. Paris: Presses universitaire de France, 1995, p. 27.

17 Lors de l'écriture de cet article, l'association Kawenga, territoires numériques existait encore. Mais, depuis juillet 2013, elle a cessé ses activités. 
Technology, il la positionne comme un lieu, acteur de l'innovation et de l'expérimentation dans la création numérique. L'utilisation de ce terme laisse entrevoir la volonté de rapprocher, au sein d'une même structure culturelle, les acteurs de la création artistique numérique avec ceux de l'innovation technologique:

Ces laboratoires, qu'ils soient universitaires ou industriels, artistiques ou citoyens, sont des lieux où les acteurs de la création numérique, artistes, ingénieurs, chercheurs, designers, médiateurs culturels, croisent réflexions et pratiques, entre recherche et production, science et nouvelles technologies, art et société, dans l'esprit de la culture libre ${ }^{18}$.

L'exemple le plus évident chez Kawenga de cette volonté d'inscrire les arts numériques dans le champ de la recherche artistique et technologique se nomme Scène(s) numérique(s). Il s'agit d'un projet de recherche et d'expérimentation sur les potentialités de la téléprésence dans le domaine artistique. Débuté depuis 2011, il consiste à organiser des cycles au cours desquels une équipe artistique et une équipe technique collaborent pour expérimenter l'élaboration d'une nouvelle forme d'écriture artistique et des solutions technologiques adaptées à sa diffusion sur les réseaux numériques. Sa première réalisation a associé l'œuvre Macadam Instinct de la compagnie du chorégraphe montpelliérain, Yann Lheureux, et une solution technique proposée par Chabalab ${ }^{19}$, une structure associative portée par Ivan Chabanaud et consacrée à l'expérimentation de dispositifs techniques qui diffusent en ligne, en temps réel, des contenus artistiques et culturels. Ces temps de recherche reposent en grande partie sur l'expérimentation artistique, une démarche inspirée de l'art expérimental, caractéristique des années 1970. Cette pratique artistique y est alors sollicitée comme activation de la recherche en arts numériques.

18 LENOBLE, Catherine. "Media Labs in Europe Mapping Places and Networks". MCD Media Labs in Europe, no 62, 2011, p. 6.

19 KAWENGA. Yann Lheureux: MacAdam Instinct scène numérique, Approche technique et artistique de la téléprésence. $<$ http://www.kawenga.org/post/2012/06/01/Yann-Lheureux-\%3A-MacAdam-Instinct-scène-numérique-Approche-
Si elle fait de Scène(s) numérique(s) un espace potentiellement productif d'œuvres d'art, la priorité est davantage accordée à la démarche de recherche portée par l'artiste plutôt qu'à la production de son œuvre d'art en tant qu'objet ou spectacle finalisé. En plus de l'expérimentation artistique, la recherche en arts numériques telle qu'elle est pratiquée dans le contexte étudié mobilise aussi l'expérimentation technologique. Dans Scène(s) numérique(s), le développement technologique n'en demeure pas moins un objectif important. Les solutions technologiques développées lors de ces cycles expérimentaux sont destinées à être réutilisées dans d'autres contextes par l'association, qui en possède un accès et les savoir-faire techniques relatifs.

Chez Kawenga, la recherche en arts numériques est conçue comme une activité inséparable de la pratique artistique et de l'expérimentation d'un processus créatif. Elle n'est pas seulement une activité créatrice d'œuvres, elle devient également productive de multiples connaissances que l'institution en tant que medialab a jugé nécessaire d'enregistrer pour être en mesure de les transmettre et les communiquer en savoirs à autrui.

\section{L'intention de communiquer la recherche en arts numériques par la documentation}

Comme Jean Davallon l'a relevé pour l'exposition, la documentation en tant que dispositif résultant d'une construction humaine émane d'une intention: "c'est-à-dire à un but ou à une volonté, de produire un effet $»^{20}$. Viviane Couzinet affirme aussi dans sa définition du dispositif info-communicationnel qu'il est issu d'une " intention sous-jacente " et " d'un effet visé $"^{21}$. En se référant au chercheur en communication Jean-Pierre Meunier, elle précise qu'un dispositif a une mission à accomplir

technique-et-artistique-de-la-téléprésence-> (consulté en décembre 2012).

20 DAVALLON, Jean. L'exposition à l'œuvre: stratégies de communication et médiation symbolique. Paris: L'Harmattan, 2000, p. 9.

21 COUZINET. «Dispositif info-communicationnels: contributions à une définition ", op. cit. p. 19. 
provenant d'une intention de départ. Dès lors, il répond à une intention, celle des acteurs qui le mettent en œuvre.

Auparavant, chez Kawenga, l'expérimentation artistique telle qu'elle était pratiquée ouvrait des réflexions, qui ne se constituaient pas en savoirs dans le sens où elles n'aboutissaient pas toujours à un résultat communicable. Les connaissances et les savoir-faire qui y étaient mobilisés demeuraient fortement attachés à l'artiste et à son équipe, ce qui apparaissait comme incompatible avec l'activité de medialab artistique qui réclame de produire des savoirs accessibles à tous. En effet, les medialabs cherchent à briser les frontières qui séparent les lieux coutumiers de la recherche et de l'innovation en privilégiant les rencontres et le travail collectif entre toutes les personnes susceptibles de contribuer à l'essor du bien commun. Dans la même logique, ils essayent de renouveler les formes de transmission des savoirs de la recherche, en les communiquant non plus par le biais des colloques ou des revues scientifiques, mais directement sur Internet, par des textes accompagnés de vidéos ou de photographies, diffusés sur les réseaux sociaux ou les blogues. Leur objectif principal est de sortir des réseaux habituels de la communication scientifique pour que le savoir soit librement accessible à tous. Cette orientation du projet Kawenga, territoires numériques, en tant que medialab, a alors favorisé la refonte de ses activités en termes de ressources, initialement dirigées vers l'organisation et l'exploitation d'un centre de documentation, réunissant des ouvrages spécialisés sur la création et les cultures numériques. L'activité de medialab a induit la nécessité par rapport à l'activité de création de rendre compte des connaissances et des savoir-faire mobilisés et développés par les artistes lors de l'exécution de leur processus créatif. Cette volonté s'est appliquée aussi bien aux projets accueillis dans le cadre de Scène(s) numérique(s) qu'à ceux accueillis en résidence dans les locaux de l'association. En effet, depuis plusieurs années, l'association possède un "pôle création " qui propose aux artistes un accompagnement en termes de conseils d'écriture, de production, de diffusion et de développement de solutions technologiques adaptées à leurs projets. Certains accompagnements aboutissent à des partenariats de production avec l'appui des aides publiques à la création (aides régionales, DICRéAM ${ }^{22}$, appels à projets...), qui se concrétisent par l'accueil d'artistes en résidences. Tout comme pour Scène(s) numérique(s), les projets soutenus sont choisis pour leurs aspects expérimentaux et innovants. Le concept de medialab incitant la structure à se positionner comme un lieu de recherche, c'est-à-dire productive de savoirs communicables, a conduit l'institution à expérimenter un dispositif documentaire qui puisse rendre les activités créatives menées productrices de savoirs transférables à tous.

L'intention communicationnelle du dispositif réside alors dans cette volonté de rendre compte des connaissances et des savoir-faire mobilisés et développés par les artistes lors de l'exécution de leur processus créatif.

\section{La mise en situation dialogale comme moteur du dispositif documentaire de Kawenga}

Les artistes se situent très souvent dans l'action et le faire, car ils vivent et pratiquent la création. Il apparaît difficile pour eux de pouvoir rendre compte d'un processus de création alors que celui-ci est vécu de manière implicite et en temps réel. Pour recueillir les connaissances, les techniques et les savoir-faire qu'ils développent, le documentaliste de Kawenga les met en situation de réflexion de manière à ce qu'ils prennent le recul nécessaire pour réfléchir à leurs pratiques et les analyser. Dans ces situations dialogales, l'artiste et son équipe
22 Dispositif pour la création artistique mutimédia: il s'agit d'un dispositif national de financement spécifique visant à soutenir le développement, la production et la diffusion d'œuvres expérimentales appartenant à la création artistique multimédia et numérique. Ce fonds d'aide est administré par le Centre national du cinéma et de l'image animée. 
se trouvent dans une posture communicationnelle qui les incite à formuler des énoncés, dans lesquels ils sont invités à exprimer clairement les connaissances et les techniques qu'ils mobilisent pendant le processus créatif et à les expliciter de manière suffisamment détaillée pour que le documentaliste puisse les recueillir. Enregistrés et retranscrits, ces énoncés lui permettent par la suite d'identifier le contenu informationnel de la recherche en arts numériques et de le fixer dans un document tertiaire, le Cahier de résidence. Il existe plusieurs situations dialogales au cœur du dispositif documentaire étudié: l'entretien semi-directif, le workshop, la présentation publique et la Convergence. Chacune possède des modalités particulières rassemblant différents énonciateurs (artistes, collaborateurs, techniciens, chercheurs, documentaliste et publics).

L'entretien semi-directif permet de centrer le discours des artistes interrogés autour de différents thèmes définis préalablement à partir des informations transmises par eux dans leur dossier de demande d'aides à la résidence.

Ces informations permettent au documentaliste de constituer un guide d'entretien centré sur l'émergence du projet artistique, sur ses principaux concepts et idées, sur ses principaux médias et médiums utilisés, sur les outils et les composantes technologiques sur lesquels il repose. Ces entretiens sont menés par le documentaliste avec l'artiste et chaque membre de son équipe. Ils sont réalisés séparément les uns des autres. En l'obligeant à formaliser ce que l'artiste contemporain est en train de faire, ces discussions initiées par l'entretien l'aident bien souvent dans son processus d'écriture. Elles lui permettent de faire un bilan réflexif après chaque phase de travail intensive. Ces entretiens permettent de recueillir des informations à un moment précis du processus créatif et en constituent une sorte de captation de ce moment, comme peut le faire, par exemple, la photographie. Ils sont réalisés régulièrement tout au long du processus créatif de manière à rendre compte de son déroulement. Dans le cas

23 MASSU, Isabelle. L'iconopathe. <http://www.iconopathe.net/> (consulté en décembre 2012). de la résidence de l'artiste Isabelle Massu, étalée sur deux années pour son projet L'iconopathe ${ }^{23}$, plusieurs entretiens semi-directifs se sont succédés, en plus des autres situations dialogales. L'éloignement de ces entretiens les uns par rapport aux autres a facilité la retranscription par le documentaliste du déroulement du processus expérimenté.

Accessible aux artistes, le workshop est un atelier au cours duquel l'artiste communique aux participants par la pratique son processus créatif. Artistes et participants se trouvent alors en situation d'échange et de collaboration active autour des connaissances, des techniques ou des savoir-faire mobilisés lors du processus créatif. Ces discussions engendrées sont importantes, car elles permettent de recueillir de nombreuses informations sur l'exécution du processus créatif. En effet, elles sont enregistrées par le documentaliste, qui assiste en tant qu'observateur au déroulement du workshop. Dans le cadre du projet Scène(s) numérique(s) et sa première mise en œuvre autour du spectacle de danse Macadam Instinct du chorégraphe Yann Lheureux, un workshop a été organisé en juin $2012^{24}$, au cours duquel les équipes artistique et technique ont transmis leur savoir-faire à des stagiaires. Macadam Instinct est un spectacle de danse qui a lieu au même moment, dans deux endroits différents, et qui repose sur un dispositif scénique composé de caméras de surveillance et d'écrans géants installés sur chacune des deux scènes. Par exemple, Yann Lheureux a déjà réalisé une représentation simultanée à l'île de la Réunion et à Dakar au Sénégal. Pendant le workshop, les stagiaires se sont partagés en deux groupes, un chez Kawenga et l'autre dans l'atelier du chorégraphe. Ils ont pu comprendre le fonctionnement du dispositif technologique en le manipulant. Ils ont piloté eux-mêmes l'interface de gestion à distance des caméras. Et, surtout, ils ont pu éprouver par eux-mêmes les enjeux artistiques liés à ces nouveaux dispositifs techniques. De son côté, le documentaliste a enregistré les discussions qui lui permettent,

24 KAWENGA. Yann Lheureux: Macadam Instinct scène numérique, Approche technique et artistique de la téléprésence, op.cit. 
dans une seconde étape, de bien présenter le dispositif technique expérimenté et développé par l'association et ses enjeux par rapport à la recherche en arts numériques. Le workshop favorise l'émergence d'une forme de communication entre les acteurs de l'expérimentation artistique et un public stagiaire qui, une fois recueillie et retranscrite, contribue à la pérennisation des pratiques artistiques.

La présentation publique est un autre rendez-vous ouvert à tous pendant lequel tout un chacun est invité à découvrir le projet en cours de réalisation au cour de la résidence et à échanger autour de la démarche des équipes artistique et technique. Ici encore, l'artiste formalise et communique l'ensemble de l'expérimentation artistique exécutée pendant la durée de la résidence. Dans la plupart des cas, il s'agit d'une forme communicationnelle relevant du discours, l'artiste s'exprimant devant un public. La posture de l'artiste change: alors que dans un workshop il adopte la posture de l'enseignant, dans la présentation publique, il devient le propre médiateur de sa démarche artistique et de son processus créatif.

Enfin, la Convergence est un rendez-vous public au cours duquel un artiste et un scientifique échangent sur leurs pratiques de recherche. Il s'agit de confronter la démarche d'expérimentation plastique et formelle d'artistes à la démarche de recherche du scientifique. Cette réflexion de fond avec un chercheur permet d'enrichir les réflexions abordées par l'artiste, de les contextualiser et d'ouvrir vers d'autres hypothèses de travail. Les informations recueillies pendant la Convergence permettent de ne pas figer la documentation sur le processus créatif, mais bien d'apporter une ouverture, un enrichissement vers d'autres disciplines. Lors de la résidence de l'artiste Thomas Tudoux pour son projet artistique Golem $^{25}$, la Convergence a réuni l'artiste et le chercheur en sciences de l'information et de la communication de l'Université de Caen, Hervé Le Crosnier. En se basant sur l'expérience proposée par Golem, une application pour téléphone portable qui invite son utilisateur à évaluer chaque acte de son existence, Le Crosnier et Tudoux ont évoqué les changements et les enjeux provoqués par la pénétration des technologies numériques dans la vie quotidienne de leurs utilisateurs. D'une part, cette Convergence a permis de contextualiser le propos de l'œuvre et la démarche de recherche de l'artiste dans une approche scientifique et, d'autre part, la démarche du chercheur s'est vue éclaircie par celle de l'artiste.

Les mises en situations dialogales présentées précédemment permettent de recueillir directement la parole de l'artiste et de ses collaborateurs. Dans le dispositif documentaire étudié, il s'agit dans la plupart des cas de situations communicationnelles reposant sur le dialogue, compris comme une forme de conversation, des questions des réponses entre plusieurs interlocuteurs. Mais il peut aussi s'agir de formes discursives relevant davantage du monologue. Les énoncés recueillis ne sont jamais exploités par le dispositif documentaire pour eux-mêmes, comme des identités uniques et isolées. Au contraire, le dispositif documentaire met les énoncés en interaction les uns avec les autres au sein même du Cahier de résidence, qui a pour objectif d'en communiquer les contenus informationnels.

\section{Les marques d'une énonciation polyphonique}

L'intention communicationnelle du dispositif documentaire de Kawenga se cristallise dans l'objet éditorial que constitue le Cahier de résidence.

Résultat final du dispositif documentaire, il a pour but la mémorisation de l'expérimentation artistique. Il s'agit plus exactement de formaliser, structurer et capitaliser le retour d'expériences des artistes et de leurs équipes dans la manière dont ils ont mené l'expérimentation de leur processus créatif. Ce document écrit est considéré comme tertiaire, c'est-àdire qu'il compile et explicite l'ensemble des 
contenus informationnels présents dans des documents secondaires, recueillis et produits préalablement tout au long du déroulement de l'expérimentation artistique, elle-même perçue comme document primaire à étudier et à enregistrer. En effet, le dispositif documentaire développé par Kawenga consiste à analyser le processus créatif expérimenté au sein de la résidence selon une approche de l'informationdocumentation ${ }^{26}$. Il est alors envisagé comme un document de la même manière que la pionnière de la documentation Suzanne Briet a attribué le statut de document à l'antilope d'Afrique, capturée dans un zoo $^{27}$. Celle-ci se transforme en document au moment où elle devient un objet d'étude, pour un destinataire. En suivant ce schéma, le processus créatif de l'expérimentation artistique peut être considéré comme un document, car il constitue l'objet d'étude des acteurs de la recherche en arts numériques: «le document existe parce qu'un usager en a besoin pour prouver ou expliquer quelque chose, comprendre, apprendre, produire, etc. ${ }^{28} "$. Les documents secondaires comportent des informations sur ce document primaire. Recueillis ou produits lors des premières étapes du dispositif documentaire analysé, ils appartiennent à plusieurs typologies: captations audiovisuelles, dossiers, entretiens retranscrits... Dans un parcours incessant d'aller-retour vers ces derniers, le documentaliste cherche à rendre lisibles les informations sur le processus créatif et l'expérimentation artistique qui y sont contenues. Le documentaliste passe du statut de récepteur des documents secondaires à celui de producteur du document tertiaire. Il engage un véritable travail de fond qui l'oblige à saisir tous les enjeux transposés par l'expérimentation du processus créatif. Pour cela, il mobilise une technique s'apparentant au principe de l'analyse documentaire qui consiste en

\begin{abstract}
une opération intellectuelle visant à identifier les informations contenues dans un document ou un ensemble de documents et à les exprimer sans interprétation ni critique, sous une forme concise et précise telle qu'un résultat d'indexation, un résumé, un extrait. Le but est de permettre la mémorisation, le repérage, la diffusion ultérieure des informations ou du document source ${ }^{29}$.
\end{abstract}

Son rôle est bien de représenter l'information récoltée dans ces documents secondaires sous une forme condensée, organisée et lisible.

Il réalise un travail de mise en forme éditoriale, mais aussi de fond, mobilisant ses compétences d'observation, de lecture et d'écriture.

Le concept opératoire d'énonciation éditoriale, développé par Emmanuel Souchier, est mobilisé au sein du présent article pour étudier le Cahier de résidence en prenant en compte la dimension visuelle de l'information écrite de manière à dévoiler des marques d'énonciation, qui relèvent de " l'infra-ordinaire " et qui cherchent à « rester cachées $»^{30}$. En apparence, le Cahier de résidence contient une énonciation, neutre et impersonnelle, caractérisée par l'utilisation de la troisième personne. L'extrait du Cahier de résidence du projet S.U.N. illustre l'utilisation de ce style d'écriture:
Jusqu'à ce projet, Young-Ho Nam avait l'habitude de réaliser elle-même l'ensemble du processus de création, dont elle avait une maîtrise absolue. Au cours d'une première étape, elle créait sa chorégraphie avec les danseurs tandis que le travail de musique et de lumière s'ef- fectuait lors d'une seconde étape lorsque l'écriture chorégraphique était terminée. L'échange entre le musicien, l'éclaira- giste et la chorégraphe était réduit à son strict minimum. Avec le projet S.U.N.,

29 BOULOGNE, Arlette. Vocabulaire de la documentation. Paris: ADBS, 2004 ; cité par COURBIÈRES, Caroline. "L'analyse documentaire: éléments d'organisation des connaissances ». In. GARDIÈS, Approche de l'information-documentation: concepts fondateurs, op. cit., p. 151. 30 SOUCHIER, "L'image du texte, pour une théorie de l'énonciation éditoriale ", op. cit., p. 137-145.
26 GARDIÈS, Cécile. Approche de l'information-documentation: concepts fondateurs. Toulouse: Cépaduès, 2011. 27 BRIET, Suzanne. Qu'est-ce que la documentation? Paris: Éditions documentaires industrielles et techniques, 1951. 28 GARDIÈS. Approche de l'information-documentation: concepts fondateurs, op. cit., p. 42. 
Young-Ho Nam a voulu remettre en cause la manière dont elle avait l'habitude de mener son travail artistique. Cette envie d'utiliser les capteurs et le numérique l'a conduite à repenser le mode de création de son spectacle en privilégiant un processus collectif $^{31}$.

Cependant, en portant un regard plus attentif, certaines marques d'énonciation éditoriale présentes dans le Cahier de résidence laissent entrevoir la présence d'une énonciation polyphonique. Tout d'abord, sous chacune des photographies, le nom de la personne qui a pris le cliché est mentionné, en petits caractères. Plusieurs noms apparaissent. Cette marque d'énonciation éditoriale révèle que les photographies, qui constituent des documents secondaires du système documentaire étudié, proviennent de plusieurs contributeurs. La note d'intention de l'artiste, quant à elle, est une citation directe de la parole de la chorégraphe (ce qui est confirmé par l'utilisation de la première personne):

Depuis quelques années, je réfléchis à l'apport des technologies numériques dans mon travail chorégraphique. Avec le projet S.U.N., un travail en équipe, avec des spécialistes des nouvelles technologies, permettra d'ouvrir une voie vers la compréhension du mystère des mouvements invisibles du corps ${ }^{32}$.

Entre guillemets, la note d'intention se termine par cette phrase mise en italique: "Young-Ho Nam, extrait de son dossier artistique " ${ }^{33}$. Cette dernière phrase et l'utilisation de la première personne indiquent que la note d'intention provient d'un autre document secondaire réuni par le système documentaire, le dossier artistique rédigé en partie par la chorégraphe Young-Ho Nam lors de sa demande de résidence. De même, cette fois-ci au cœur du texte du Cahier de résidence, la mise entre guillemets de plusieurs expressions comme "le souffle nécessaire $^{34}$ » ou « chercheuse du corps ${ }^{35}$ " révèle que

31 KAWENGA. Territoires numériques. Les Cahiers de résidence, S.U.N. (Seon universel numérique), Young-Ho Nam, Résidence à Kawenga, 2010-2011, p. 43. 32 Id., p. 9. ces expressions sont des citations de la parole de la chorégraphe, ces dernières provenant directement des retranscriptions de l'entretien mené par le documentaliste avec la danseuse.

Ces différentes marques d'énonciation éditoriale présentes dans le Cahier de résidence constituent des traces fortement dissimulées de l'énonciation polyphonique, qui caractérise les processus communicationnels qui traversent le dispositif documentaire de Kawenga.

\section{Une énonciation documentaire plurielle}

Si, par son travail d'écriture du Cahier de résidence, le documentaliste apparaît comme l'acteur majeur de l'énonciation documentaire, qui régit le dispositif documentaire examiné dans cet article, il n'en constitue pas le seul protagoniste, comme le laissent suggérer les marques d'énonciation polyphoniques dévoilées précédemment. En effet, l'analyse du dispositif documentaire comme dispositif info-communicationnel a permis d'y déterminer différents énonciateurs.

Dans un premier temps, tous les documentstraces mobilisent un auteur - le producteur du document - et sont porteurs d'une intention communicationnelle initiale. Les documentstraces sont qualifiés de documents secondaires, car ils comportent des informations sur le processus créatif réalisé pendant l'expérimentation artistique, lui-même considéré comme le document primaire. Ils deviennent utiles lorsqu'ils sont analysés par le documentaliste, qui les explique et les rend intelligibles. Prises tout au long de la résidence, les captations audiovisuelles, par exemple, possèdent une valeur descriptive, qui peut être transformée en information lorsqu'elle est explicitée par le documentaliste dans le Cahier de résidence. D'autres documents-traces peuvent être réunis, comme le dossier artistique déposé par l'artiste lors de sa demande de résidence, des comptes

33 Id., p. 10.

34 Ibid.

35 Ibid. 
rendus de réunions ou encore des documents administratifs. Tous ces documents secondaires sont détournés de leur fonction informative première pour être mis au service de la compréhension de l'expérimentation artistique. Dans la même logique, d'autres documents peuvent être rassemblés selon les techniques de la recherche documentaire, particulièrement à partir d'Internet. Ils ont cette fois-ci comme attribution un statut de ressource, permettant d'en connaître davantage sur l'artiste, un de ses anciens projets, une de ses références, ou sur une solution technique utilisée.

Dans un second temps, toutes les mises en situations dialogales comportent elles aussi une multitude d'énonciateurs. L'entretien semi-directif, par exemple, en comporte deux, le documentaliste et l'artiste interrogé, comme la Convergence, qui réunit l'artiste et un chercheur. La présentation publique, qui adopte plutôt la forme d'un discours, mobilise généralement un énonciateur unique, l'artiste contemporain. Le workshop, quant à lui, met en scène un nombre plus important d'énonciateurs. Par exemple, pour le workshop de Scène(s) numérique(s) autour du projet Macadam Instinct, les énonciateurs présents étaient le chorégraphe Yann Lheureux, le technicien Ivan Chabanaud et le régisseur Éric Hennaut, ainsi qu'une dizaine de participants. Les échanges ont eu lieu entre l'ensemble de ces protagonistes. Selon le processus créatif expérimenté, le nombre d'énonciateurs au sein du dispositif documentaire de Kawenga peut largement augmenter, notamment s'il induit un travail collectif et collaboratif rassemblant des compétences différentes appartenant à plusieurs disciplines. Le projet expérimental S.U.N. ${ }^{36}$ autour de l'utilisation de capteurs corporels placés sur les danseurs, dirigés par la chorégraphe coréenne Young-Ho Nam, a réuni par exemple plus de treize protagonistes.

La mise en œuvre du dispositif documentaire de Kawenga repose sur une pluralité d'énonciateurs et d'énoncés, fixés sur différents documents, produits par diverses mises en situations communicationnelles, qui se croisent et se répètent tout au long de la résidence artistique. Les processus communicationnels qui en sont issus s'entremêlent et s'entrecroisent les uns aux autres au sein même du dispositif documentaire.

\section{Conclusion}

Le dispositif documentaire étudié est hybride dans le sens où il est productif à la fois de documents secondaires et tertiaires. Les deux le sont à partir du document primaire que constitue le processus créatif expérimenté lors d'une résidence organisée par l'association. En effet, les premières étapes sont productives de documents secondaires, qu'il s'agisse de la phase concernant l'assemblage de documents-traces ou de la mise en situation dialogale de l'artiste contemporain, tandis que la seconde étape, qui consiste à élaborer et rédiger le Cahier de résidence, cherche à rendre lisibles les informations présentes dans les divers documents secondaires produits préalablement. Le rôle de celui-ci est de faciliter l'accès aux informations scientifiques de la recherche en arts numériques, c'est-à-dire à l'expérimentation artistique et à son processus créatif. Le dispositif documentaire analysé est donc porteur de savoirs scientifiques. S'il constitue de manière évidente un dispositif informationnel dans le sens où il donne accès à des informations sur le processus créatif de la recherche en arts numériques, il est également un dispositif communicationnel, car il permet l'énonciation des connaissances et des savoir-faire issus de la pratique artistique. Cette énonciation apparaît comme étant plurielle et partagée entre divers énonciateurs, qui interviennent dans les nombreux processus communicationnels traversant le dispositif documentaire. L'analyse déployée ici démontre l'existence d'un glissement de la documentation vers la communication scientifique. Le dévoilement des processus de communication et de l'énonciation polyphonique 
du dispositif documentaire de Kawenga atteste de la capacité de la documentation, comprise à la fois comme un ensemble de documents et comme une activité, de devenir un outil pour communiquer la recherche en arts numériques.

\section{Si l'usage de l'énonciation documentaire} polyphonique est mobilisé par les acteurs de la recherche en arts numériques pour dépasser l'éclatement des résultats engendrés par le travail collectif et multidisciplinaire de la recherche en ce domaine, il laisse entrevoir une autre difficulté engendrée cette fois-ci par le partage de l'énonciation des savoirs scientifiques entre les protagonistes de la recherche et le documentaliste. En effet, même si le documentaliste s'engage à respecter leur parole en essayant de coller le plus précisément à celle-ci, minimisant au maximum la valeur interprétative de sa réécriture, le travail de traduction des propos ne peut s'opérer sans un processus interprétatif coproducteur de sens: «Quelle que soit la nature du document à traiter, la situation de communication engage le documentaliste à re-construire le contenu informationnel du document en procédant à son analyse interprétative ${ }^{37}$. " Cette difficulté rejoint les préoccupations de l'artiste-chercheur Pierre Gosselin, qui estime que la communication scientifique de la recherche en art devrait être mise en énonciation directement par l'artiste:

Qu'il soit motivé par le désir de parler de sa pratique artistique, des œuvres ou de tout autre objet, c'est en tenant compte de son point de vue particulier de praticien que le chercheur en pratique artistique doit le faire. Le discours résultant de sa recherche devrait naturellement traduire le point de vue qui lui est propre. Et pour cela, il faut trouver sa voix. Trouver sa voix, c'est à mon sens le défi que pose actuellement au praticien la recherche en pratique artistique $^{38}$.
Ce paradoxe persistant au sein du dispositif documentaire étudié mérite d'être éclairci, notamment par une étude plus approfondie des éléments de négociation, qui délimitent le partage de la parole scientifique entre le documentaliste et les protagonistes de la recherche en arts numériques.

Le présent article s'est concentré sur un exemple précis vécu par le chercheur-praticien dans une démarche de recherche par l'action. Il serait intéressant d'analyser d'autres pratiques documentaires appartenant à d'autres contextes de la recherche en arts numériques, sous l'angle de la situation d'énonciation, qui viendraient en éléments comparatifs enrichir et valider les résultats issus de cette première étude.
38 GOSSELIN, Pierre et Éric LE COGUIEC. $\mathrm{La}$ Recherche création: Pour une compréhension de la recherche en pratique artistique. Montréal: Presses de l'Université du Québec, 2006, p. 30. 
Stéphane Bellin, translated by Olga Gorban

\section{Documenting and communicating digital art research through the dialogism of the contemporary artist.}

The proposed paper provides an unprecedented review of the dialogism, designed as a mechanism, an engine of a documentation system of digital art research. The latter is an activity extremely hybrid, characterised by its capacity to produce simultaneously a work of art, technical devices and theoretical knowledge about the art and the technologies. ${ }^{1}$ Faced with a number of issues and the spectacular results, valued in different academic fields and by many contributors ${ }^{2}$, the performers of digital art research are trying to invent the forms of its scientific communication. Moreover, in the context of the University and the Graduate Art Schools, many artists-researchers note that the research in arts does not give the immediate results, for it is largely based on the hardly observable practices. The articulation between the theory and the practice leads to the emergence of scientific communication in order to shape a word, which, according to Pierre Gosselin, a professor within the Quebec context who introduced the concept research creation, is situated "in-between", between the doing of the creative practice and the verb of the theoretical practice of the research ${ }^{3}$.

The novelty of the proposed approach in this paper consists in analysing the documentation as "in-between", i.e. as a means of putting in words and of collecting knowledge and expertise that come into light by the creative process. The information-documentation approach enables to consider it as a document, a carrier of the information to transmit, since it performs within the info-communicational scheme which registers it and communicates it. The advantage of the dialogism is that it incites the contemporary artists and their teams to think in a way that allows them to step back to analyse their practices and to communicate them.

The argumentation of this paper is based on the analysis of the documentation system and its different dialogical situations ${ }^{4}$, developed as part of artistic projects in Kawenga, digital territory. Located in Montpellier (France), this cultural structure is an artistic medialab, an experimental ground for the arts and the digital cultures, symptomatic of the convergence of the technological innovation and the contemporary artistic

1 FOURMENTRAUX, Jean-Paul. "L'art à l'épreuve de la recherche. Profession et identité artistique en mutation ». In. BIANCHINI, Samuel (dir.) Recherche et Création. Nancy: Les Editions du parc, 2009, p.80.

2 FOURMENTRAUX, Jean-Paul. Artistes de laboratoires, Recherche et création à l'ère numérique. Paris: Hermann, 2011, p.75.

3 BURNS, L. Sophia. "La parole de l'artiste chercheur ". In. GOSSELIN, Pierre et Éric LE COEGUIEC (dir.).

La Recherche création, pour une compréhension de la recherche en pratique artistique. Québec: Presses de l'Université du Québec, 2006, p.63.

4 Each of them has a particular modality grouping different enunciators. 
creativity. The study of this documentation system calls on two concept-tools: that of info-communicational scheme ${ }^{5}$, which enables to look at it through its various forms (technical, socio-organisational and socio-economic), and that of editorial enunciation ${ }^{6}$, which enables to conceive the plurality of enunciation of its dialogical situations.

The proposed analysis in this paper shows that the documentation is shifting to the scientific communication. The disclosing of plural editorial's enunciation marks proves the capacity of the documentation, perceived both as a set of documents and an activity, to become a tool for digital art research communication. 